\title{
LIMIT THEOREMS FOR A DIFFUSION PROCESS WITH A ONE-SIDED BROWNIAN POTENTIAL
}

\author{
KIYOSHI KAWAZU,* Yamaguchi University \\ YUKI SUZUKI, ${ }^{* *}$ Keio University
}

\begin{abstract}
We consider a diffusion process $X(t)$ with a one-sided Brownian potential starting from the origin. The limiting behavior of the process as time goes to infinity is studied. For each $t>0$, the sample space describing the random potential is divided into two parts, $\tilde{A}_{t}$ and $\tilde{B}_{t}$, both having probability $\frac{1}{2}$, in such a way that our diffusion process $X(t)$ exhibits quite different limiting behavior depending on whether it is conditioned on $\tilde{A}_{t}$ or on $\tilde{B}_{t}$ $(t \rightarrow \infty)$. The asymptotic behavior of the maximum process of $X(t)$ is also investigated. Our results improve those of Kawazu, Suzuki, and Tanaka (2001).
\end{abstract}

Keywords: Random environment; diffusion process; occupation time

2000 Mathematics Subject Classification: Primary 60J60; 60K37

\section{Introduction}

In [4] a diffusion process $\{X(t), t \geq 0\}$ with a one-sided Brownian potential was studied, and it was shown that the limit distribution of $t^{-1 / 2} X(t)$ as $t \rightarrow \infty$ exists and is given by

$$
\frac{1}{2} \sqrt{\frac{2}{\pi}} \mathrm{e}^{-x^{2} / 2} \mathrm{~d} x+\frac{1}{2} \delta_{0}(\mathrm{~d} x),
$$

the support being $[0, \infty)$. The long-time behavior of $X(t)$ is diffusive (in the sense that a limit distribution exists under the Brownian scaling) with probability $\frac{1}{2}$ and subdiffusive with the remaining probability $\frac{1}{2}$.

In this paper we treat the same model and give much more precise statements. In fact we prove, among other things, that $(\log t)^{-2} X(t)$ has a limit distribution with probability $\frac{1}{2}$; for the precise meaning of this, see Theorem 1.5.

Let us describe our model, following [4]. We denote by $\mathbb{W}$ the space of continuous functions $w$ defined on $\mathbb{R}$ and vanishing identically on $[0, \infty)$. Let $\mathrm{P}$ be the Wiener measure on $\mathbb{W}$, namely the probability measure on $\mathbb{W}$ such that $\{w(-x), x \geq 0, \mathrm{P}\}$ is a Brownian motion with time parameter $x$. By $\Omega$ we denote the space of real-valued, continuous functions defined on $[0, \infty)$. For $\omega \in \Omega$, we write $X(t) \equiv X(t, \omega) \equiv \omega(t)$, the value of $\omega$ at $t$. For $w \in \mathbb{W}$ and $x_{0} \in \mathbb{R}$, we let $\mathrm{P}_{w}^{x_{0}}$ be the probability measure on $\Omega$ such that $\left\{X(t), t \geq 0, \mathrm{P}_{w}^{x_{0}}\right\}$ is a diffusion process with generator

$$
\mathcal{L}_{w}=\frac{1}{2} \mathrm{e}^{w(x)} \frac{\mathrm{d}}{\mathrm{d} x}\left(\mathrm{e}^{-w(x)} \frac{\mathrm{d}}{\mathrm{d} x}\right),
$$

Received 22 February 2005; revision received 1 September 2006.

* Postal address: Department of Mathematics, Faculty of Education, Yamaguchi University, Yoshida, Yamaguchi, 753-8513, Japan.

** Postal address: School of Medicine, Keio University, Hiyoshi, Kouhoku-ku, Yokohama, 223-8521, Japan.

Email address: yuki@hc.cc.keio.ac.jp 
starting from $x_{0}$. We define the probability measure $\mathcal{P}^{x_{0}}$ on $\mathbb{W} \times \Omega$ by

$$
\mathcal{P}^{x_{0}}(\mathrm{~d} w \mathrm{~d} \omega)=\mathrm{P}(\mathrm{d} w) \mathrm{P}_{w}^{x_{0}}(\mathrm{~d} \omega) .
$$

We regard $\left\{X(t), t \geq 0, \mathcal{P}^{x_{0}}\right\}$ as a process defined on the probability space ( $\mathbb{W} \times \Omega, \mathcal{P}^{x_{0}}$ ) and call it a diffusion process with a one-sided Brownian potential. Our aim is to clarify the limiting behavior of $\left\{X(t), t \geq 0, \mathcal{P}^{0}\right\}$ as $t \rightarrow \infty$.

For the case in which $w(x)$ does not vanish identically for $x \geq 0$ or, more precisely, the case in which $\{w(x), x \geq 0, \mathrm{P}\}$ and $\{w(-x), x \geq 0, \mathrm{P}\}$ are independent Brownian motions, the corresponding diffusion process, $\left\{X(t), t \geq 0, \mathcal{P}^{x_{0}}\right\}$, was introduced by Brox [1] and Schumacher [5] as a diffusion analogue of Sinai's random walk [6]. In [1] and [5] it was proved that $\left\{(\log t)^{-2} X(t), t \geq 0, \mathcal{P}^{0}\right\}$ has a nondegenerate limit distribution.

We begin by presenting the result of [4]. Let $\mathcal{M}$ be the space of probability laws on $\Omega$ and let $\rho$ be the Prokhorov metric on $\mathcal{M}$. Let $\left\{X(t), t \geq 0, \mathcal{P}^{0}\right\}$ be a diffusion process with a one-sided Brownian potential. Set

$$
X_{\lambda}(t)=\lambda^{-1 / 2} X(\lambda t), \quad t \geq 0,
$$

for a constant $\lambda>0$, and denote by $\mathrm{P}_{\lambda}(w) \in \mathcal{M}$ the probability law of the process $\left\{X_{\lambda}(t)\right.$, $\left.t \geq 0, \mathrm{P}_{w}^{0}\right\}$. Also, denote by $\mathrm{P}_{\mathrm{N}} \in \mathcal{M}$ the probability law of the identically vanishing process, and by $\mathrm{P}_{\mathrm{R}} \in \mathcal{M}$ the probability law of the reflecting Brownian motion on $[0, \infty)$ starting from 0 .

Theorem 1.1. ([4].) For any $\varepsilon$ such that $0<\varepsilon<\rho\left(\mathrm{P}_{\mathrm{N}}, \mathrm{P}_{\mathrm{R}}\right) / 2$,

$$
\begin{aligned}
& \lim _{\lambda \rightarrow \infty} \mathrm{P}\left\{\rho\left(\mathrm{P}_{\lambda}(w), \mathrm{P}_{\mathrm{N}}\right)<\varepsilon\right\}=\frac{1}{2}, \\
& \lim _{\lambda \rightarrow \infty} \mathrm{P}\left\{\rho\left(\mathrm{P}_{\lambda}(w), \mathrm{P}_{\mathrm{R}}\right)<\varepsilon\right\}=\frac{1}{2} .
\end{aligned}
$$

Our present results (namely Theorems 1.2 and 1.3, stated below) imply Theorem 1.1. To state the theorems, we introduce some notation.

For $w \in \mathbb{W}$ and $x_{0} \in \mathbb{R}$, the diffusion process $\left\{X(t), t \geq 0, \mathrm{P}_{w}^{x_{0}}\right\}$ can be constructed from a Brownian motion via time change and scale change [3, p. 165]. See [4] for the explicit representation of this diffusion process. The scale function of the process is given by

$$
S(x)=\int_{0}^{x} \mathrm{e}^{w(y)} \mathrm{d} y, \quad x \in \mathbb{R} .
$$

If $S(x) \rightarrow-\infty$ (as $x \rightarrow-\infty$ ), then the diffusion process $\left\{X(t), t \geq 0, \mathrm{P}_{w}^{x_{0}}\right\}$ is recurrent and, hence, conservative. By restricting the whole space $\mathbb{W}$ to the set of $w$ s satisfying $S(x) \rightarrow$ $-\infty$ (as $x \rightarrow-\infty)$, which still has P-measure 1, we may assume that the diffusion process $\left\{X(t), t \geq 0, \mathrm{P}_{w}^{x_{0}}\right\}$ is recurrent for any $w$.

For $\omega \in \Omega$, we write

$$
a(t) \equiv a(t, \omega)=\int_{0}^{t} \mathbf{1}_{(0, \infty)}(X(s)) \mathrm{d} s, \quad t \geq 0,
$$

where $\mathbf{1}_{A}$ denotes the indicator function of the (generic) set $A$. Then, for any $w \in \mathbb{W}$ and $x_{0} \in \mathbb{R}$, we have

$$
\mathrm{P}_{w}^{x_{0}}\left\{\lim _{t \rightarrow \infty} a(t)=\infty\right\}=1,
$$


since the diffusion process $\left\{X(t), t \geq 0, \mathrm{P}_{w}^{x_{0}}\right\}$ is recurrent. In what follows, we reduce $\Omega$ so that it equals the set of $\omega$ s satisfying $a(t) \rightarrow \infty$ (as $t \rightarrow \infty)$. For $\lambda>0$ and $\omega \in \Omega$, we let

$$
a_{\lambda}(t) \equiv a_{\lambda}(t, \omega)=\int_{0}^{t} \mathbf{1}_{(0, \infty)}\left(X_{\lambda}(s)\right) \mathrm{d} s, \quad t \geq 0 .
$$

Since $a_{\lambda}(t)=\lambda^{-1} a(\lambda t) \rightarrow \infty($ as $t \rightarrow \infty)$, we can define

$$
a_{\lambda}^{-1}(t)=\inf \left\{s>0: a_{\lambda}(s)>t\right\}, \quad t \geq 0,
$$

the right-continuous inverse function of $a_{\lambda}(t)$. We also let

$$
G_{\lambda}(t)=X_{\lambda}\left(a_{\lambda}^{-1}(t)\right), \quad t \geq 0 .
$$

Then $\left\{G_{\lambda}(t), t \geq 0, \mathrm{P}_{w}^{0}\right\}$ is a reflecting Brownian motion on $[0, \infty)$ starting from 0 .

For $w \in \mathbb{W}$ and $a \in \mathbb{R}$, we let

$$
\sigma(a) \equiv \sigma(a, w)=\sup \{x<0: w(x)=a\},
$$

and introduce two subsets $A$ and $B$ of $\mathbb{W}$ as follows:

$$
\begin{aligned}
& A=\left\{w \in \mathbb{W}: \sigma\left(\frac{1}{2}\right)>\sigma\left(-\frac{1}{2}\right)\right\}, \\
& B=\left\{w \in \mathbb{W}: \sigma\left(\frac{1}{2}\right)<\sigma\left(-\frac{1}{2}\right)\right\} .
\end{aligned}
$$

Each of these subsets has P-measure $\frac{1}{2}$. For $w \in \mathbb{W}$ and $\lambda>0$, we define $w_{\lambda} \in \mathbb{W}$ by

$$
w_{\lambda}(x)=\lambda^{-1} w\left(\lambda^{2} x\right), \quad x \in \mathbb{R} .
$$

Then

$$
\left\{w_{\lambda}, \mathrm{P}\right\} \stackrel{\mathrm{D}}{=}\{w, P\}
$$

where ' $\stackrel{\mathrm{D}}{=}$ denotes equality in distribution. For each $\lambda>0$, we also introduce two further subsets $A_{\lambda}$ and $B_{\lambda}$ of $\mathbb{W}$ as follows:

$$
\begin{aligned}
& A_{\lambda}=\left\{w \in \mathbb{W}: w_{\lambda} \in A\right\}, \\
& B_{\lambda}=\left\{w \in \mathbb{W}: w_{\lambda} \in B\right\} .
\end{aligned}
$$

Each of these also has P-measure $\frac{1}{2}$, by (1.2).

In the following theorems, $\mathrm{P}\{\cdots \mid \cdot\}$ denotes the conditional probability. We write $\tilde{A}_{\lambda}=$ $A_{\log \lambda}$ and $\tilde{B}_{\lambda}=B_{\log \lambda}$.

Theorem 1.2. For any $T>0$ and $\varepsilon>0$,

$$
\lim _{\lambda \rightarrow \infty} \mathrm{P}\left\{\mathrm{P}_{w}^{0}\left\{\sup _{0 \leq t \leq T}\left|X_{\lambda}(t)-G_{\lambda}(t)\right|<\varepsilon\right\}>1-\varepsilon \mid \tilde{A}_{\lambda}\right\}=1 .
$$

For $w \in \mathbb{W}$, we let

$$
\begin{aligned}
& \zeta \equiv \zeta(w)=\sup \left\{x<0: w(x)-\min _{x \leq y \leq 0} w(y)=1\right\}, \\
& M \equiv M(w)= \begin{cases}\sigma\left(\frac{1}{2}\right) & \text { if } w \in A, \\
\zeta(w) & \text { if } w \in B,\end{cases} \\
& V \equiv V(w)=\min _{x \geq M} w(x) .
\end{aligned}
$$

We also define $b \equiv b(w)$ in $(M, 0)$ by $w(b)=V$. Note that $b$ is determined uniquely by $w$ (P-almost surely). 
Theorem 1.3. For any $\varepsilon>0$,

$$
\lim _{t \rightarrow \infty} \mathrm{P}\left\{\mathrm{P}_{w}^{0}\left\{\left|(\log t)^{-2} X(t)-b\left(w_{\log t}\right)\right|<\varepsilon\right\}>1-\varepsilon \mid \tilde{B}_{t}\right\}=1 .
$$

To state the result on the maximum process of $X(t)$, we let

$$
H(w)=\max _{M \leq x \leq 0} w(x) .
$$

Note that $H(w)=\frac{1}{2}$ if $w \in A$ and $0<H(w)<\frac{1}{2}$ if $w \in B$.

Theorem 1.4. For any $\varepsilon>0$,

$$
\lim _{t \rightarrow \infty} \mathcal{P}^{0}\left\{\left|\frac{\log \max _{0 \leq s \leq t} X(s)}{\log t}-H\left(w_{\log t}\right)\right|>\varepsilon\right\}=0 .
$$

Our present results, together with those of [4], immediately imply the following theorem.

Theorem 1.5. In each of the following instances, the distribution of $\tilde{X}_{t}$ under $\mathcal{P}^{0}$ tends to a limit distribution as $t \rightarrow \infty$, as described.

- $\tilde{X}_{t}=t^{-1 / 2} X(t)$ : limit distribution is $\mu_{\mathrm{I}}(\mathrm{d} x)=\frac{1}{2} \sqrt{2 / \pi} \mathrm{e}^{-x^{2} / 2} \mathrm{~d} x+\frac{1}{2} \delta_{0}(\mathrm{~d} x)$; support is $[0, \infty)$.

- $\tilde{X}_{t}=(\log t)^{-2} X(t)$ : limit distribution is $\mu_{\mathrm{II}}(\mathrm{d} x)=\mathrm{P}\{(b \in \mathrm{d} x) \cap B\}+\frac{1}{2} \delta_{\infty}(\mathrm{d} x)$; support is $(-\infty, 0) \cup\{\infty\}$.

- $\tilde{X}_{t}=t^{-1 / 2} \max _{0 \leq s \leq t} X(s)$ : limit distribution is

$$
\mu_{\mathrm{III}}(\mathrm{d} x)=\frac{1}{2} \mathrm{P}_{\mathrm{R}}\left\{\max _{0 \leq s \leq 1} X(s) \in \mathrm{d} x\right\}+\frac{1}{2} \delta_{0}(\mathrm{~d} x) ;
$$

support is $[0, \infty)$.

- $\tilde{X}_{t}=\log \left(\max _{0 \leq s \leq t} X(s)\right) / \log t$ : limit distribution is $\mu_{\mathrm{IV}}(\mathrm{d} x)=\mathrm{P}\{H \in \mathrm{d} x\}$; support is $\left(0, \frac{1}{2}\right]$.

- $\tilde{X}_{t}=(\log t)^{-2} \min _{0 \leq s \leq t} X(s)$ : limit distribution is $\mu_{\mathrm{V}}(\mathrm{d} x)=\mathrm{P}\{M \in \mathrm{d} x\}$; support is $(-\infty, 0)$.

Moreover, the Laplace transforms of the distributions of $b, H$, and $M$ appearing in the definitions of $\mu_{\mathrm{II}}, \mu_{\mathrm{IV}}$, and $\mu_{\mathrm{V}}$ are as follows. For $\xi>0$,

$$
\begin{aligned}
\mathrm{E}\left[\mathrm{e}^{\xi b}, B\right] & =\frac{\sinh (\sqrt{2 \xi} / 2)}{\sqrt{2 \xi} \cosh \sqrt{2 \xi}}, \\
\mathrm{E}\left[\mathrm{e}^{\xi H}, A\right] & =\frac{1}{2} \mathrm{e}^{\xi / 2}, \\
\mathrm{E}\left[\mathrm{e}^{\xi H}, B\right] & =\int_{0}^{1 / 2} \mathrm{e}^{\xi x} \mathrm{~d} x, \\
\mathrm{E}\left[\mathrm{e}^{\xi M}, A\right] & =\frac{\sinh (\sqrt{2 \xi} / 2)}{\sinh \sqrt{2 \xi}}, \\
\mathrm{E}\left[\mathrm{e}^{\xi M}, B\right] & =\frac{\sinh (\sqrt{2 \xi} / 2)}{(\sinh \sqrt{2 \xi})(\cosh \sqrt{2 \xi})} .
\end{aligned}
$$

Here $\mathrm{E}[\cdot, A]$ denotes the expectation with respect to $\mathrm{P}$ on the set $A$. 
Let $\eta(t, x)$ be the local time at $x$ of the Brox-Schumacher diffusion process. Hu and Shi [2] showed that, for any $x \in \mathbb{R}$,

$$
\frac{\log \eta(t, x)}{\log t} \stackrel{\mathrm{D}}{\rightarrow} U \wedge \hat{U}, \quad t \rightarrow \infty
$$

where ' $\stackrel{\text { D }}{\rightarrow}$ ' denotes convergence in distribution and $U$ and $\hat{U}$ are independent random variables uniformly distributed in $(0,1)$.

For our diffusion process $\left\{X(t), t \geq 0, \mathcal{P}^{0}\right\}$ with a one-sided Brownian potential, Z. Shi (private communication (2000)) informed us that the same method based on the second RayKnight theorem as in [2] can be used to show that

$$
\frac{\log a(t)}{\log t} \stackrel{\mathrm{D}}{\rightarrow} 1 \wedge(2 U), \quad t \rightarrow \infty,
$$

where $U$ is a random variable uniformly distributed in $(0,1)$.

In Section 3 we investigate the asymptotic behavior of the occupation time $a(t)$ as $t \rightarrow \infty$.

\section{Preliminaries}

For $\lambda>0, w \in \mathbb{W}$, and $x_{0} \in \mathbb{R}$, let $\mathrm{P}_{\lambda w}^{x_{0}}$ be the probability measure on $\Omega$ such that $\left\{X(t), t \geq 0, \mathrm{P}_{\lambda w}^{x_{0}}\right\}$ is a diffusion process with generator

$$
\mathcal{L}_{\lambda w}=\frac{1}{2} \mathrm{e}^{\lambda w(x)} \frac{\mathrm{d}}{\mathrm{d} x}\left(\mathrm{e}^{-\lambda w(x)} \frac{\mathrm{d}}{\mathrm{d} x}\right),
$$

starting from $x_{0}$. Denote by $\mathrm{E}_{\lambda w}^{x_{0}}$ the expectation with respect to $\mathrm{P}_{\lambda w}^{x_{0}}$. The following lemma was proved in [1].

Lemma 2.1. ([1].) For any $\lambda>0$ and $w \in \mathbb{W}$,

$$
\left\{X(t), t \geq 0, \mathrm{P}_{\lambda w_{\lambda}}^{0}\right\} \stackrel{\mathrm{D}}{=}\left\{\lambda^{-2} X\left(\lambda^{4} t\right), t \geq 0, \mathrm{P}_{w}^{0}\right\} .
$$

In preparation for the proofs of Theorem 1.2 and Theorem 1.3, we present the following theorems.

Theorem 2.1. Let $\mu \equiv \mu(\lambda)=\lambda^{1 / 4} \log \lambda$. Then, for any $T>0$ and $\varepsilon>0$,

$$
\lim _{\lambda \rightarrow \infty} \mathrm{P}\left\{\mathrm{P}_{\mu w_{\mu}}^{0}\left\{\sup _{0 \leq t \leq T}|X(t)-G(t)|<\varepsilon\right\}>1-\varepsilon \mid A_{\log \lambda}\right\}=1,
$$

where $G(t)=X\left(a^{-1}(t)\right)$ and $a^{-1}(t)=\inf \{s>0: a(s)>t\}$, the right-continuous inverse function of a $(t)$ defined in (1.1).

Theorem 2.2. Let $r$ be a real-valued function of $\lambda>0$ such that $r(\lambda) \rightarrow 1($ as $\lambda \rightarrow \infty)$. Then there exists a subset $B^{\#}$ of $B$, with $\mathrm{P}\left\{B \backslash B^{\#}\right\}=0$, such that, for any $w \in B^{\#}$ and $\varepsilon>0$,

$$
\lim _{\lambda \rightarrow \infty} \mathrm{P}_{\lambda w}^{0}\left\{\left|X\left(\mathrm{e}^{\lambda r(\lambda)}\right)-b(w)\right|<\varepsilon\right\}=1 .
$$

We remark that to prove Theorem 2.2 it is enough to show the following proposition. 
Proposition 2.1. There exists a subset $B^{\#}$ of $B$, with $\mathrm{P}\left\{B \backslash B^{\#}\right\}=0$, such that for any $w \in B^{\#}$ the following holds: there exists a $\delta>0$ such that, for any $r_{1}$ and $r_{2}$ satisfying $1-\delta<r_{1}<$ $r_{2}<1+\delta$ and any $\varepsilon>0$,

$$
\lim _{\lambda \rightarrow \infty} \inf _{r \in\left[r_{1}, r_{2}\right]} \mathrm{P}_{\lambda w}^{0}\left\{\left|X\left(\mathrm{e}^{\lambda r}\right)-b(w)\right|<\varepsilon\right\}=1 .
$$

In Section 4 we prove Theorem 2.1 and Theorem 1.2, in Section 5 we prove Proposition 2.1 and Theorem 1.3, and in Section 6 we prove Theorem 1.4.

\section{Asymptotic behavior of the occupation time $a(t)$ as $t \rightarrow \infty$}

In this section we investigate the limiting behavior of $\left\{t^{-1} a(t), t \geq 0, \mathcal{P}^{0}\right\}$ as $t \rightarrow \infty$. To do so, we need two lemmas. The first, Lemma 3.1, which is needed for the proof of Theorem 2.1, will be proved in Section 4.

Lemma 3.1. There exists a subset $A^{\#}$ of $A$, with $\mathrm{P}\left\{A \backslash A^{\#}\right\}=0$, such that, for any $w \in A^{\#}$ and $T>0$,

$$
\lim _{\lambda \rightarrow \infty} \mathrm{E}_{\lambda w}^{0}\left[\frac{1}{T \mathrm{e}^{\lambda}} \int_{0}^{T \mathrm{e}^{\lambda}} \mathbf{1}_{(0, \infty)}(X(s)) \mathrm{d} s\right]=1 .
$$

The second lemma, Lemma 3.2, can be obtained from Proposition 2.1.

Lemma 3.2. There exists a subset $B^{\#}$ of $B$, with $\mathrm{P}\left\{B \backslash B^{\#}\right\}=0$, such that, for any $w \in B^{\#}$,

$$
\lim _{\lambda \rightarrow \infty} \mathrm{E}_{\lambda w}^{0}\left[\frac{1}{\mathrm{e}^{\lambda}} \int_{0}^{\mathrm{e}^{\lambda}} \mathbf{1}_{(-\infty, 0)}(X(s)) \mathrm{d} s\right]=1 .
$$

The main result in this section is as follows.

Theorem 3.1. For any $\varepsilon>0$,

$$
\begin{array}{r}
\lim _{t \rightarrow \infty} \mathrm{P}\left\{\mathrm{P}_{w}^{0}\left\{\frac{1}{t} \int_{0}^{t} \mathbf{1}_{(0, \infty)}(X(s)) \mathrm{d} s>1-\varepsilon\right\}>1-\varepsilon \mid \tilde{A}_{t}\right\}=1, \\
\lim _{t \rightarrow \infty} \mathrm{P}\left\{\mathrm{P}_{w}^{0}\left\{\frac{1}{t} \int_{0}^{t} \mathbf{1}_{(0, \infty)}(X(s)) \mathrm{d} s<\varepsilon\right\}>1-\varepsilon \mid \tilde{B}_{t}\right\}=1 .
\end{array}
$$

Proof. We prove (3.2); we can prove (3.3) in the same way by using Lemma 3.2. By Lemma 3.1 we have, for $w \in A^{\# \text {, }}$

$$
\lim _{t \rightarrow \infty} \mathrm{E}_{(\log t) w}^{0}\left[\frac{1}{t} \int_{0}^{t} \mathbf{1}_{(0, \infty)}(X(s)) \mathrm{d} s\right]=1 .
$$

Here $A^{\#}$ is a subset of $A$ with $\mathrm{P}\left\{A \backslash A^{\#}\right\}=0$. Therefore, it follows that

$$
\lim _{t \rightarrow \infty} \mathrm{P}\left\{\mathrm{P}_{(\log t) w}^{0}\left\{\frac{1}{t} \int_{0}^{t} \mathbf{1}_{(0, \infty)}(X(s)) \mathrm{d} s>1-\varepsilon\right\}>1-\varepsilon \mid A\right\}=1
$$

for any $\varepsilon>0$. Moreover, (1.2) and Lemma 2.1 imply that

$$
\lim _{t \rightarrow \infty} \mathrm{P}\left\{\mathrm{P}_{w}^{0}\left\{\frac{1}{t} \int_{0}^{t} \mathbf{1}_{(0, \infty)}\left(X\left((\log t)^{4} s\right)\right) \mathrm{d} s>1-\varepsilon\right\}>1-\varepsilon \mid A_{\log t}\right\}=1 .
$$


Changing the variable of the integral in (3.4) yields

$$
\lim _{u \rightarrow \infty} \mathrm{P}\left\{\mathrm{P}_{w}^{0}\left\{\frac{1}{u} \int_{0}^{u} \mathbf{1}_{(0, \infty)}(X(s)) \mathrm{d} s>1-\varepsilon\right\}>1-\varepsilon \mid A_{\log t}\right\}=1,
$$

where $t \equiv t(u)$ is determined by $u=t(\log t)^{4}$.

Let us prove that

$$
\lim _{u \rightarrow \infty} \mathrm{P}\left\{A_{\log t(u)} \ominus A_{\log u}\right\}=0,
$$

where $A_{1} \ominus A_{2}=\left(A_{1} \cap A_{2}^{\mathrm{c}}\right) \cup\left(A_{1}^{\mathrm{c}} \cap A_{2}\right)$ for sets $A_{1}$ and $A_{2}$. We note that

$$
r=\frac{\log t(u)}{\log u} \rightarrow 1 \quad \text { as } u \rightarrow \infty .
$$

Using the fact that $w_{\lambda_{1} \lambda_{2}}=\left(w_{\lambda_{1}}\right)_{\lambda_{2}}$ for $\lambda_{1}, \lambda_{2}>0$, we have

$$
\begin{aligned}
\mathrm{P}\left\{A_{\log t(u)} \ominus A_{\log u}\right\} & =\mathrm{E}\left[\left|\mathbf{1}_{A}\left(w_{\log t(u)}\right)-\mathbf{1}_{A}\left(w_{\log u}\right)\right|\right] \\
& =\mathrm{E}\left[\left|\mathbf{1}_{A}\left(\left(w_{\log u}\right)_{r}\right)-\mathbf{1}_{A}\left(w_{\log u}\right)\right|\right],
\end{aligned}
$$

where $\mathrm{E}$ denotes the expectation with respect to $\mathrm{P}$. By (1.2), the right-hand side of (3.8) is equal to

$$
\mathrm{E}\left[\left|\mathbf{1}_{A}\left(w_{r}\right)-\mathbf{1}_{A}(w)\right|\right],
$$

which converges to 0 as $u \rightarrow \infty$, due to (3.7). This proves (3.6). From (3.6) it follows that $\mathrm{P}\left\{\cdots \mid A_{\log t(u)}\right\} \rightarrow 1($ as $u \rightarrow \infty)$ is equivalent to $\mathrm{P}\left\{\cdots \mid A_{\log u}\right\} \rightarrow 1($ as $u \rightarrow \infty)$. Hence, by (3.5), we obtain (3.2).

Corollary 3.1. The probability distribution of $t^{-1} \int_{0}^{t} \mathbf{1}_{(0, \infty)}(X(s)) \mathrm{d} s$ under $\mathcal{P}^{0}$ converges to $\frac{1}{2} \delta_{0}+\frac{1}{2} \delta_{1}$ as $t \rightarrow \infty$.

\section{Proofs of Theorem 2.1 and Theorem 1.2}

In this section we prove Theorem 2.1 and Theorem 1.2. First we introduce a lemma from [4]. For $\omega \in \Omega$, let

$$
\tau(a) \equiv \tau(a, \omega)=\inf \{t>0: X(t)=a\}, \quad a \in \mathbb{R} .
$$

Lemma 4.1. ([4].) Let $w \in \mathbb{W}$ and $a<0$. Assume that $w(a)>w(x)$ for all $x>a$. Then, for any $\varepsilon>0$,

$$
\lim _{\lambda \rightarrow \infty} \mathrm{P}_{\lambda w}^{0}\left\{\mathrm{e}^{\lambda(J-\varepsilon)}<\tau(a)<\mathrm{e}^{\lambda(J+\varepsilon)}\right\}=1,
$$

where $J=\max \left\{J_{0}, 2 w(a)\right\}$ and $J_{0}=w(a)-\min \{w(x): x \geq a\}$.

Next we prove Lemma 3.1.

Proof of Lemma 3.1. Let $w \in A$. In this case we note that $V>-\frac{1}{2}$. First we choose $r_{0}$ and $r_{1}$ satisfying $-V<r_{0}<r_{1}<\frac{1}{2}$. We then see that

$$
\lim _{\lambda \rightarrow \infty} \mathrm{P}_{\lambda w}^{0}\left\{\tau\left(\mathrm{e}^{r_{0} \lambda}\right)<\tau\left(\sigma\left(r_{1}\right)\right)\right\}=\lim _{\lambda \rightarrow \infty} \frac{\int_{\sigma\left(r_{1}\right)}^{0} \mathrm{e}^{\lambda w(x)} \mathrm{d} x}{\int_{\sigma\left(r_{1}\right)}^{0} \mathrm{e}^{\lambda w(x)} \mathrm{d} x+\mathrm{e}^{r_{0} \lambda}}=1,
$$


since $\lim _{\lambda \rightarrow \infty} \lambda^{-1} \log \int_{\sigma\left(r_{1}\right)}^{0} \mathrm{e}^{\lambda w(x)} \mathrm{d} x=r_{1}>r_{0}$. Moreover, an application of Lemma 4.1 with $a=\sigma\left(r_{1}\right)$ gives

$$
\lim _{\lambda \rightarrow \infty} \mathrm{P}_{\lambda w}^{0}\left\{\mathrm{e}^{\lambda\left(2 r_{1}-\varepsilon\right)}<\tau\left(\sigma\left(r_{1}\right)\right)<\mathrm{e}^{\lambda\left(2 r_{1}+\varepsilon\right)}\right\}=1
$$

for any $\varepsilon>0$. Combining (4.1) and (4.2) yields

$$
\lim _{\lambda \rightarrow \infty} \mathrm{P}_{\lambda w}^{0}\left\{\tau\left(\mathrm{e}^{r_{0} \lambda}\right)<\mathrm{e}^{\lambda \theta}\right\}=1 \quad \text { for some } \theta \in(0,1) .
$$

Next we choose a $\rho>\frac{1}{2}$ satisfying

$$
\min \left\{w(x): \sigma(\rho) \leq x \leq \sigma\left(\frac{1}{2}\right)\right\}>V
$$

(Note that the set of $w \in A$ for which there is no $\rho>\frac{1}{2}$ satisfying (4.4) is P-negligible.) By applying Lemma 4.1 with $a=\sigma(\rho)$, we have

$$
\lim _{\lambda \rightarrow \infty} \mathrm{P}_{\lambda w}^{0}\left\{\mathrm{e}^{\lambda(2 \rho-\varepsilon)}<\tau(\sigma(\rho))<\mathrm{e}^{\lambda(2 \rho+\varepsilon)}\right\}=1
$$

for any $\varepsilon>0$. Therefore, for any $T>0$,

$$
\lim _{\lambda \rightarrow \infty} \mathrm{P}_{\lambda w}^{0}\left\{\tau(\sigma(\rho))>T \mathrm{e}^{\lambda}\right\}=1 .
$$

Now we define $m_{\lambda w}$, a probability measure on $I_{\lambda}=\left[\sigma(\rho), \mathrm{e}^{r_{0} \lambda}\right]$, by

$$
m_{\lambda w}(E)=\frac{\int_{E \cap[\sigma(\rho), 0]} \mathrm{e}^{-\lambda w(x)} \mathrm{d} x+\int_{E \cap\left(0, \mathrm{e}^{r_{0} \lambda}\right]} \mathrm{d} x}{\int_{\sigma(\rho)}^{0} \mathrm{e}^{-\lambda w(x)} \mathrm{d} x+\mathrm{e}^{r_{0} \lambda}}
$$

for any Borel set $E$ in $I_{\lambda}$. This is the invariant probability measure for the reflecting $\mathcal{L}_{\lambda w}$-diffusion process on $I_{\lambda}$. Note that

$$
\lim _{\lambda \rightarrow \infty} m_{\lambda w}\left(\left(0, \mathrm{e}^{r_{0} \lambda}\right]\right)=\lim _{\lambda \rightarrow \infty} \frac{\mathrm{e}^{r_{0} \lambda}}{\int_{\sigma(\rho)}^{0} \mathrm{e}^{-\lambda w(x)} \mathrm{d} x+\mathrm{e}^{r_{0} \lambda}}=1
$$

since $\lim _{\lambda \rightarrow \infty} \lambda^{-1} \log \int_{\sigma(\rho)}^{0} \mathrm{e}^{-\lambda w(x)} \mathrm{d} x=-V<r_{0}$.

By the comparison theorem for one-dimensional diffusion processes, for $\lambda>0$ we can construct diffusion processes $\left\{Y_{\lambda}(t), t \geq 0\right\}$ and $\left\{Z_{\lambda}(t), t \geq 0\right\}$, on a probability space $(\tilde{\Omega}, \tilde{\mathrm{P}})$, with the following properties:

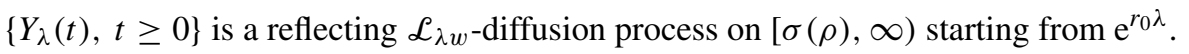

$\left\{Z_{\lambda}(t), t \geq 0\right\}$ is a reflecting $\mathcal{L}_{\lambda w}$-diffusion process on $I_{\lambda}$ with initial distribution $m_{\lambda w}$.

$$
\tilde{\mathrm{P}}\left\{Y_{\lambda}(t) \geq Z_{\lambda}(t) \text { for all } t \geq 0\right\}=1 \text {. }
$$

Since $\left\{Z_{\lambda}(t), t \geq 0\right\}$ is a stationary process with invariant probability measure $m_{\lambda w}$, by (4.6) we have

$$
\lim _{\lambda \rightarrow \infty} \tilde{\mathrm{E}}\left[\frac{1}{T \mathrm{e}^{\lambda}} \int_{0}^{T \mathrm{e}^{\lambda}} \mathbf{1}_{(0, \infty)}\left(Z_{\lambda}(s)\right) \mathrm{d} s\right]=1
$$


for any $T>0$. Here $\tilde{\mathrm{E}}$ denotes the expectation with respect to $\tilde{\mathrm{P}}$. Moreover, (4.8) and (4.9) imply that

$$
\lim _{\lambda \rightarrow \infty} \tilde{\mathrm{E}}\left[\frac{1}{T \mathrm{e}^{\lambda}} \int_{0}^{T \mathrm{e}^{\lambda}} \mathbf{1}_{(0, \infty)}\left(Y_{\lambda}(s)\right) \mathrm{d} s\right]=1 .
$$

Let us prove (3.1). Using the strong Markov property of $\left\{X(t), t \geq 0, P_{\lambda w}^{\cdot}\right\}$, for any $T>0$ we obtain

$$
\begin{aligned}
\mathrm{E}_{\lambda w}^{0} & {\left[\frac{1}{T \mathrm{e}^{\lambda}} \int_{0}^{T \mathrm{e}^{\lambda}} \mathbf{1}_{(0, \infty)}(X(s)) \mathrm{d} s\right] } \\
& \geq \mathrm{E}_{\lambda w}^{0}\left[\frac{1}{T \mathrm{e}^{\lambda}} \int_{\tau\left(\mathrm{e}^{r_{0} \lambda}\right)}^{T \mathrm{e}^{\lambda}} \mathbf{1}_{(0, \infty)}(X(s)) \mathrm{d} s, \tau\left(\mathrm{e}^{r_{0} \lambda}\right)<\mathrm{e}^{\lambda \theta}\right] \\
& \geq \mathrm{E}_{\lambda w}^{\mathrm{e}^{r} 0^{\lambda}}\left[\frac{1}{T \mathrm{e}^{\lambda}} \int_{0}^{T \mathrm{e}^{\lambda}-\mathrm{e}^{\lambda \theta}} \mathbf{1}_{(0, \infty)}(X(s)) \mathrm{d} s\right] \mathrm{P}_{\lambda w}^{0}\left\{\tau\left(\mathrm{e}^{r_{0} \lambda}\right)<\mathrm{e}^{\lambda \theta}\right\} .
\end{aligned}
$$

Owing to (4.3), (4.5), (4.7), and (4.10), we see that the right-hand side of (4.11) converges to 1 as $\lambda \rightarrow \infty$. Hence, we obtain (3.1).

We now present three lemmas in preparation for the proof of Theorem 2.1.

Lemma 4.2. Let $\mu \equiv \mu(\lambda)=\lambda^{1 / 4} \log \lambda$. Then, for any $T>0$ and $\varepsilon>0$,

$$
\lim _{\lambda \rightarrow \infty} \mathrm{P}\left\{\mathrm{P}_{\mu w_{\mu}}^{0}\left\{\sup _{0 \leq t \leq T}|t-a(t)|<\varepsilon\right\}>1-\varepsilon \mid A_{\log \lambda}\right\}=1 .
$$

Proof. Using Chebyshev's inequality, we have

$$
\begin{aligned}
\mathrm{P}\left\{\mathrm{P}_{\mu w_{\mu}}^{0}\left\{\sup _{0 \leq t \leq T}|t-a(t)| \geq \varepsilon\right\} \geq \varepsilon, A_{\log \lambda}\right\} & =\mathrm{P}\left\{\mathrm{P}_{\mu w_{\mu}}^{0}\{T-a(T) \geq \varepsilon\} \geq \varepsilon, A_{\log \lambda}\right\} \\
& \leq \mathrm{P}\left\{\frac{1}{\varepsilon} \mathrm{E}_{\mu w_{\mu}}^{0}[T-a(T)] \geq \varepsilon, A_{\log \lambda}\right\} .
\end{aligned}
$$

By Lemma 2.1, we have

$$
\begin{aligned}
\mathrm{E}_{\mu w_{\mu}}^{0}[a(T)] & =\mathrm{E}_{\mu w_{\mu}}^{0}\left[\int_{0}^{T} \mathbf{1}_{(0, \infty)}(X(s)) \mathrm{d} s\right] \\
& =\mathrm{E}_{w}^{0}\left[\int_{0}^{T} \mathbf{1}_{(0, \infty)}\left(X_{\lambda(\log \lambda)^{4}}(s)\right) \mathrm{d} s\right] \\
& =\mathrm{E}_{w}^{0}\left[\frac{1}{\lambda} \int_{0}^{T \lambda} \mathbf{1}_{(0, \infty)}\left(X_{(\log \lambda)^{4}}(s)\right) \mathrm{d} s\right] \\
& =\mathrm{E}_{(\log \lambda) w_{\log \lambda}}^{0}\left[\frac{1}{\lambda} \int_{0}^{T \lambda} \mathbf{1}_{(0, \infty)}(X(s)) \mathrm{d} s\right] \\
& =\mathrm{E}_{(\log \lambda) w_{\log \lambda}}^{0}\left[\frac{1}{\lambda} a(T \lambda)\right] .
\end{aligned}
$$

Therefore, the right-hand side of (4.12) is equal to

$$
\mathrm{P}\left\{\frac{1}{\varepsilon} \mathrm{E}_{(\log \lambda) w_{\log \lambda}}^{0}\left[T-\frac{1}{\lambda} a(T \lambda)\right] \geq \varepsilon, A_{\log \lambda}\right\}=\mathrm{P}\left\{\frac{1}{\varepsilon} \mathrm{E}_{(\log \lambda) w}^{0}\left[T-\frac{1}{\lambda} a(T \lambda)\right] \geq \varepsilon, A\right\} .
$$


This probability converges to 0 as $\lambda \rightarrow \infty$, since

$$
\lim _{\lambda \rightarrow \infty} \mathrm{E}_{(\log \lambda) w}^{0}\left[\frac{1}{T \lambda} a(T \lambda)\right]=1 \quad \text { for } w \in A^{\#}
$$

by Lemma 3.1. Here $A^{\#}$ is a subset of $A$ with $\mathrm{P}\left\{A \backslash A^{\#}\right\}=0$. Hence, we obtain Lemma 4.2.

Lemma 4.3. Let $\mu \equiv \mu(\lambda)=\lambda^{1 / 4} \log \lambda$. Then, for any $T>0$ and $\varepsilon>0$,

$$
\lim _{\lambda \rightarrow \infty} \mathrm{P}\left\{\mathrm{P}_{\mu w_{\mu}}^{0}\left\{\sup _{0 \leq t \leq T}|G(t)-G(a(t))|<\varepsilon\right\}>1-\varepsilon \mid A_{\log \lambda}\right\}=1 .
$$

Proof. We note that, for any $w \in \mathbb{W}$ and $\lambda>0,\left\{G(t), t \geq 0, \mathrm{P}_{\mu w_{\mu}}^{0}\right\}$ is a reflecting Brownian motion on $[0, \infty)$ starting from 0 . Since, with probability 1 , Brownian sample paths are locally Hölder continuous with exponent $\gamma$, for every $\gamma \in\left(0, \frac{1}{2}\right)$, we obtain Lemma 4.3 from Lemma 4.2.

The following lemma plays an important role in the proof of Theorem 2.1.

Lemma 4.4. Let $f$ be a real-valued, continuous function of $t \geq 0$ with $f(0)=0$, and let

$$
\alpha(t)=\int_{0}^{t} \mathbf{1}_{(0, \infty)}(f(s)) \mathrm{d} s .
$$

Take $T>0$ and assume that there exists a $T_{1}>T$ such that $\alpha\left(T_{1}\right)>T$. Define the rightcontinuous inverse function of $\alpha(t)$ by

$$
\alpha^{-1}(t)=\inf \{s>0: \alpha(s)>t\}, \quad 0 \leq t \leq T,
$$

and let

$$
g(t)=f\left(\alpha^{-1}(t)\right), \quad 0 \leq t \leq T .
$$

Then

$$
|g(t)-f(t)|=|g(t)-g(\alpha(t))|-\min \{f(t), 0\}, \quad 0 \leq t \leq T .
$$

Proof. First assume that $\alpha^{-1}(\alpha(t))=t$. In this case we notice that $f(t) \geq 0$ and

$$
|g(t)-f(t)|=\left|g(t)-f\left(\alpha^{-1}(\alpha(t))\right)\right|=|g(t)-g(\alpha(t))|,
$$

which establishes (4.13).

Next assume that $\alpha^{-1}(\alpha(t)) \neq t$. In this case $\alpha^{-1}(\alpha(t))>t$. Moreover, $f \leq 0$ on the interval $\left[t, \alpha^{-1}(\alpha(t))\right]$ and $f\left(\alpha^{-1}(\alpha(t))\right)=0$, i.e. $g(\alpha(t))=0$. Noting that $g(t) \geq 0$, we obtain

$$
|g(t)-f(t)|=|g(t)|+|f(t)|=|g(t)-g(\alpha(t))|-\min \{f(t), 0\} .
$$

This completes the proof of the lemma.

Proof of Theorem 2.1. By virtue of Lemma 4.4, we have

$$
\begin{aligned}
& \mathrm{P}\left\{\mathrm{P}_{\mu w_{\mu}}^{0}\left\{\sup _{0 \leq t \leq T}|G(t)-X(t)| \geq \varepsilon\right\} \geq \varepsilon, A_{\log \lambda}\right\} \\
& \leq \mathrm{P}\left\{\mathrm{P}_{\mu w_{\mu}}^{0}\left\{\sup _{0 \leq t \leq T}|G(t)-G(a(t))| \geq \frac{\varepsilon}{2}\right\} \geq \frac{\varepsilon}{2}, A_{\log \lambda}\right\} \\
& \quad+\mathrm{P}\left\{\mathrm{P}_{\mu w_{\mu}}^{0}\left\{\inf _{0 \leq t \leq T} X(t) \leq-\frac{\varepsilon}{2}\right\} \geq \frac{\varepsilon}{2}, A_{\log \lambda}\right\} .
\end{aligned}
$$


The first term on the right-hand side of (4.14) converges to 0 as $\lambda \rightarrow \infty$, due to Lemma 4.3. The second term is bounded above by

$$
\mathrm{P}\left\{\mathrm{P}_{\mu w_{\mu}}^{0}\left\{\inf _{0 \leq t \leq T} X(t) \leq-\frac{\varepsilon}{2}\right\} \geq \frac{\varepsilon}{2}\right\}=\mathrm{P}\left\{\mathrm{P}_{\mu w}^{0}\left\{\inf _{0 \leq t \leq T} X(t) \leq-\frac{\varepsilon}{2}\right\} \geq \frac{\varepsilon}{2}\right\},
$$

which converges to 0 as $\lambda \rightarrow \infty$, by [4, Lemma 4.2]. This completes the proof of Theorem 2.1.

Proof of Theorem 1.2. By combining Theorem 2.1 and Lemma 2.1, we have

$$
\lim _{\lambda \rightarrow \infty} \mathrm{P}\left\{\mathrm{P}_{w}^{0}\left\{\sup _{0 \leq t \leq T}\left|X_{\mu^{4}}(t)-G_{\mu^{4}}(t)\right|<\varepsilon\right\}>1-\varepsilon \mid A_{\log \lambda}\right\}=1
$$

or, equivalently,

$$
\lim _{v \rightarrow \infty} \mathrm{P}\left\{\mathrm{P}_{w}^{0}\left\{\sup _{0 \leq t \leq T}\left|X_{v}(t)-G_{v}(t)\right|<\varepsilon\right\}>1-\varepsilon \mid A_{\log \lambda}\right\}=1,
$$

where $\lambda \equiv \lambda(v)$ is determined by $v=\lambda(\log \lambda)^{4}$. We obtain Theorem 1.2 by the same argument as in the proof of Theorem 3.1.

\section{Proofs of Proposition 2.1 and Theorem 1.3}

In this section we prove Proposition 2.1 and Theorem 1.3. We begin by introducing a lemma due to Brox [1]. Let $w \in \mathbb{W}$ and $\alpha<m<\beta<0$. We call a triple of negative numbers $\Delta=(\alpha, m, \beta)$ a valley of $w$ if the following conditions are satisfied.

(i) $w(\alpha)>w(x)>w(m)$ for all $x \in(\alpha, m)$ and $w(\beta)>w(x)>w(m)$ for all $x \in(m, \beta)$.

(ii) $w(\alpha)-w(m)>H_{\beta, m}:=\sup \{w(y)-w(x): m<y<x<\beta\}$ and $w(\beta)-w(m)>$ $H_{\alpha, m}:=\sup \{w(y)-w(x): \alpha<x<y<m\}$.

For a valley $\Delta=(\alpha, m, \beta)$, we call $D(\Delta)=\{w(\alpha)-w(m)\} \wedge\{w(\beta)-w(m)\}$ the depth of $\Delta$ and $A(\Delta)=H_{\beta, m} \vee H_{\alpha, m}$ the inner directed ascent of $\Delta$. A valley $\Delta=(\alpha, m, \beta)$ is said to contain $x_{0}$ if $\alpha<x_{0}<\beta$.

Lemma 5.1. ([1].) Let $w \in \mathbb{W}$ and let $\Delta=(\alpha, m, \beta)$ be a valley of $w$ containing $x_{0}$. Then, for any $r_{1}$ and $r_{2}$ satisfying $A(\Delta)<r_{1}<r_{2}<D(\Delta)$ and any $\varepsilon>0$,

$$
\lim _{\lambda \rightarrow \infty} \inf _{r \in\left[r_{1}, r_{2}\right]} \mathrm{P}_{\lambda w}^{x_{0}}\left\{\left|X\left(\mathrm{e}^{\lambda r}\right)-m\right|<\varepsilon\right\}=1 .
$$

Let us now prove Proposition 2.1.

Proof of Proposition 2.1. Let $w \in B$. In this case $M=\zeta$ and $V<-\frac{1}{2}$. Let

$$
V^{\prime} \equiv V^{\prime}(w)=\max _{x \geq b} w(x)
$$

and define $b^{\prime} \equiv b^{\prime}(w)$ in $(b, 0)$ by $w\left(b^{\prime}\right)=V^{\prime}$. Note that $b^{\prime}$ is determined uniquely by $w$ (P-almost surely).

First we consider the case $V^{\prime}-V>1$. Let

$$
c \equiv c(w)=\sup \left\{x<b^{\prime}: w(x)=0\right\}
$$


and define $\tilde{w} \in \mathbb{W}$ by

$$
\tilde{w}(x)= \begin{cases}w(x) & \text { for } x \geq c, \\ -x+c & \text { for } x<c .\end{cases}
$$

We can choose a $c^{\prime}<c$ satisfying

$$
\begin{gathered}
\tilde{w}\left(b^{\prime}\right)<\tilde{w}\left(c^{\prime}\right)<\frac{1}{2}, \\
\tilde{J}:=\left\{\tilde{w}\left(c^{\prime}\right)-\min _{c^{\prime} \leq x \leq 0} \tilde{w}(x)\right\} \vee 2 \tilde{w}\left(c^{\prime}\right)<1 .
\end{gathered}
$$

An application of Lemma 4.1 with $a=c^{\prime}$ yields

$$
\lim _{\lambda \rightarrow \infty} \mathrm{P}_{\lambda \tilde{w}}^{0}\left\{\tau\left(c^{\prime}\right)<\mathrm{e}^{\lambda(\tilde{J}+\varepsilon)}\right\}=1
$$

for any $\varepsilon>0$. Since

$$
\mathrm{P}_{\lambda \tilde{w}}^{0}\left\{\tau\left(c^{\prime}\right)<\mathrm{e}^{\lambda(\tilde{J}+\varepsilon)}\right\} \leq \mathrm{P}_{\lambda \tilde{w}}^{0}\left\{\tau(c)<\mathrm{e}^{\lambda(\tilde{J}+\varepsilon)}\right\}=\mathrm{P}_{\lambda w}^{0}\left\{\tau(c)<\mathrm{e}^{\lambda(\tilde{J}+\varepsilon)}\right\},
$$

(5.1) implies that

$$
\lim _{\lambda \rightarrow \infty} \mathrm{P}_{\lambda w}^{0}\left\{\tau(c)<\mathrm{e}^{\lambda \theta_{0}}\right\}=1 \quad \text { for some } \theta_{0} \in(0,1) .
$$

On the other hand, we see that $\Delta=\left(\zeta, b, b^{\prime}\right)$ is a valley of $w$ of depth 1 containing $c$. Thus, there exists a negative number $\alpha$ such that $\alpha<\zeta$ and $\Delta^{\prime}=\left(\alpha, b, b^{\prime}\right)$ is a valley of $w$ containing $c$ with $A\left(\Delta^{\prime}\right)<1<D\left(\Delta^{\prime}\right)$. (The set of $w \in B$ for which there is no $\alpha$ satisfying this condition is P-negligible [1].) Therefore, by Lemma 5.1, there exists a $\delta_{0}>0$ such that, for any $r_{1}$ and $r_{2}$ satisfying $1-\delta_{0}<r_{1}<r_{2}<1+\delta_{0}$ and any $\varepsilon>0$,

$$
\lim _{\lambda \rightarrow \infty} \inf _{r \in\left[r_{1}, r_{2}\right]} \mathrm{P}_{\lambda w}^{\mathrm{c}}\left\{\left|X\left(\mathrm{e}^{\lambda r}\right)-b\right|<\varepsilon\right\}=1 .
$$

Using the strong Markov property of $\left\{X(t), t \geq 0, \mathrm{P}_{\lambda w}\right\}$ and (5.2) and (5.3), we obtain (2.1) for any $\delta \in\left(0, \delta_{0} \wedge\left(1-\theta_{0}\right)\right)$ in the case $V^{\prime}-V>1$.

Next we let $V^{\prime}-V<1$. In this case we note that $0<V^{\prime}<w(\zeta)<\frac{1}{2}$. Thus, we can choose a $\rho^{\prime}$ satisfying $V^{\prime}<\rho^{\prime}<w(\zeta)$, and note that $\sigma\left(\rho^{\prime}\right)<b$. Applying Lemma 4.1 with $a=\sigma\left(\rho^{\prime}\right)$ yields

$$
\lim _{\lambda \rightarrow \infty} \mathrm{P}_{\lambda w}^{0}\left\{\mathrm{e}^{\lambda\left(\rho^{\prime}-V-\varepsilon\right)}<\tau\left(\sigma\left(\rho^{\prime}\right)\right)<\mathrm{e}^{\lambda\left(\rho^{\prime}-V+\varepsilon\right)}\right\}=1
$$

for any $\varepsilon>0$. Since $\rho^{\prime}-V<1$, it follows that

$$
\lim _{\lambda \rightarrow \infty} \mathrm{P}_{\lambda w}^{0}\left\{\tau\left(\sigma\left(\rho^{\prime}\right)\right)<\mathrm{e}^{\lambda \theta_{1}}\right\}=1 \quad \text { for some } \theta_{1} \in(0,1)
$$

Also, we can choose a $\rho$ satisfying

$$
\begin{gathered}
w(\zeta)<\rho<\frac{1}{2}, \\
\min \{w(x): \sigma(\rho) \leq x \leq \zeta\}>V .
\end{gathered}
$$

(Note that the set of $w \in B$ for which there is no $\rho$ satisfying (5.5) is P-negligible.) An application of Lemma 4.1 with $a=\sigma(\rho)$ yields

$$
\lim _{\lambda \rightarrow \infty} \mathrm{P}_{\lambda w}^{0}\left\{\mathrm{e}^{\lambda(\rho-V-\varepsilon)}<\tau(\sigma(\rho))<\mathrm{e}^{\lambda(\rho-V+\varepsilon)}\right\}=1
$$


for any $\varepsilon>0$. Let $\tau_{\lambda}=\tau(\sigma(\rho)) \wedge \tau\left(e^{\lambda / 2}\right)$; we then observe that

$$
\lim _{\lambda \rightarrow \infty} \mathrm{P}_{\lambda w}^{0}\left\{\tau(\sigma(\rho))<\tau\left(\mathrm{e}^{\lambda / 2}\right)\right\}=\lim _{\lambda \rightarrow \infty} \frac{\mathrm{e}^{\lambda / 2}}{\int_{\sigma(\rho)}^{0} \mathrm{e}^{\lambda w(x)} \mathrm{d} x+\mathrm{e}^{\lambda / 2}}=1,
$$

because $\lim _{\lambda \rightarrow \infty} \lambda^{-1} \log \int_{\sigma(\rho)}^{0} \mathrm{e}^{\lambda w(x)} \mathrm{d} x=\rho<\frac{1}{2}$. By (5.6) and (5.7), for any $\varepsilon>0$ we have

$$
\lim _{\lambda \rightarrow \infty} \mathrm{P}_{\lambda w}^{0}\left\{\mathrm{e}^{\lambda(\rho-V-\varepsilon)}<\tau_{\lambda}<\mathrm{e}^{\lambda(\rho-V+\varepsilon)}\right\}=1 .
$$

Since $\rho-V>1$, for any small $\delta_{1}>0$ we may consider the process $\{X(t), 0 \leq t \leq$ $\left.\mathrm{e}^{\lambda\left(1+\delta_{1}\right)}, \mathrm{P}_{\lambda w}^{0}\right\}$ to be a reflecting $\mathcal{L}_{\lambda w}$-diffusion process on $I_{\lambda}^{\prime}=\left[\sigma(\rho), \mathrm{e}^{\lambda / 2}\right]$. We define $m_{\lambda w}^{\prime}$, a probability measure on $I_{\lambda}^{\prime}$, by

$$
m_{\lambda w}^{\prime}(E)=\frac{\int_{E \cap[\sigma(\rho), 0]} \mathrm{e}^{-\lambda w(x)} \mathrm{d} x+\int_{E \cap\left(0, \mathrm{e}^{\lambda / 2}\right]} \mathrm{d} x}{\int_{\sigma(\rho)}^{0} \mathrm{e}^{-\lambda w(x)} \mathrm{d} x+\mathrm{e}^{\lambda / 2}}
$$

for any Borel set $E$ in $I_{\lambda}^{\prime}$. This is the invariant probability measure for the reflecting $\mathcal{L}_{\lambda w}$-diffusion process on $I_{\lambda}^{\prime}$. Notice that, for any $\varepsilon>0$ satisfying $[b-\varepsilon, b+\varepsilon] \subset[\sigma(\rho), 0]$,

$$
\lim _{\lambda \rightarrow \infty} m_{\lambda w}^{\prime}((b-\varepsilon, b+\varepsilon))=\lim _{\lambda \rightarrow \infty} \frac{\int_{b-\varepsilon}^{b+\varepsilon} \mathrm{e}^{-\lambda w(x)} \mathrm{d} x}{\int_{\sigma(\rho)}^{0} \mathrm{e}^{-\lambda w(x)} \mathrm{d} x+\mathrm{e}^{\lambda / 2}}=1,
$$

since

$$
\begin{array}{r}
\lim _{\lambda \rightarrow \infty} \frac{1}{\lambda} \log \int_{b-\varepsilon}^{b+\varepsilon} \mathrm{e}^{-\lambda w(x)} \mathrm{d} x=-V>\frac{1}{2}, \\
\lim _{\lambda \rightarrow \infty} \frac{1}{\lambda} \log \int_{(b-\varepsilon, b+\varepsilon)^{\mathrm{c}} \cap[\sigma(\rho), 0]} \mathrm{e}^{-\lambda w(x)} \mathrm{d} x<-V .
\end{array}
$$

Recall that $\sigma\left(\rho^{\prime}\right)<b<0$. In the following, $\varepsilon>0$ is chosen to be small enough that $\sigma\left(\rho^{\prime}\right)<b-\varepsilon$ and $b+\varepsilon<0$. Let $\left\{X_{\lambda}^{\mathrm{R}}(t), t \geq 0\right\}$ be a reflecting $\mathcal{L}_{\lambda w}$-diffusion process on $I_{\lambda}^{\prime}$ with initial distribution $m_{\lambda w}^{\prime}$ defined on a probability space $(\tilde{\Omega}, \tilde{\mathrm{P}})$. This is a stationary process. From (5.8), it follows that

$$
\lim _{\lambda \rightarrow \infty} \tilde{\mathrm{P}}\left\{b-\varepsilon<X_{\lambda}^{\mathrm{R}}(0)<b+\varepsilon\right\}=1
$$

and that, for any $r_{1}$ and $r_{2}$ satisfying $0<r_{1}<r_{2}$,

$$
\lim _{\lambda \rightarrow \infty} \inf _{r \in\left[r_{1}, r_{2}\right]} \tilde{\mathrm{P}}\left\{b-\varepsilon<X_{\lambda}^{\mathrm{R}}\left(\mathrm{e}^{\lambda r}\right)<b+\varepsilon\right\}=1 .
$$

By (5.9), (5.10), and the comparison theorem for one-dimensional diffusion processes, we deduce that

$$
\begin{aligned}
& \lim _{\lambda \rightarrow \infty} \inf _{r \in\left[r_{1}, r_{2}\right]} \mathrm{P}_{\lambda w}^{b-\varepsilon}\left\{X\left(\mathrm{e}^{\lambda r}\right)<b+\varepsilon\right\}=1, \\
& \lim _{\lambda \rightarrow \infty} \inf _{r \in\left[r_{1}, r_{2}\right]} \mathrm{P}_{\lambda w}^{0}\left\{X\left(\mathrm{e}^{\lambda r}\right)>b-\varepsilon\right\}=1,
\end{aligned}
$$

for any $r_{1}$ and $r_{2}$ satisfying $0<r_{1}<r_{2}<1+\delta_{1}$. 
Now, by (5.4), we notice that

$$
\lim _{\lambda \rightarrow \infty} \mathrm{P}_{\lambda w}^{0}\left\{\tau(b-\varepsilon)<\mathrm{e}^{\lambda \theta_{1}}\right\}=1 \quad \text { for some } \theta_{1} \in(0,1) .
$$

Choose any $\delta \in\left(0, \delta_{1} \wedge\left(1-\theta_{1}\right)\right)$. Then, by the strong Markov property of $\left\{X(t), t \geq 0, \mathrm{P}_{\lambda w}\right\}$, (5.11), and (5.13), for any $r_{1}$ and $r_{2}$ satisfying $1-\delta<r_{1}<r_{2}<1+\delta$ we obtain

$$
\lim _{\lambda \rightarrow \infty} \inf _{r \in\left[r_{1}, r_{2}\right]} \mathrm{P}_{\lambda w}^{0}\left\{X\left(\mathrm{e}^{\lambda r}\right)<b+\varepsilon\right\}=1 .
$$

Combining (5.12) and (5.14) yields (2.1) for $V^{\prime}-V<1$. This completes the proof of Proposition 2.1.

Proof of Theorem 1.3. Using Lemma 2.1 and (1.2), we have

$$
\begin{aligned}
& \mathrm{P}\left\{\mathrm{P}_{w}^{0}\left\{\left|\lambda^{-2} X\left(\mathrm{e}^{\lambda}\right)-b\left(w_{\lambda}\right)\right|<\varepsilon\right\}>1-\varepsilon, B_{\lambda}\right\} \\
& \quad=\mathrm{P}\left\{\mathrm{P}_{\lambda w}^{0}\left\{\left|X\left(\mathrm{e}^{\lambda r(\lambda)}\right)-b(w)\right|<\varepsilon\right\}>1-\varepsilon, B\right\},
\end{aligned}
$$

where $r(\lambda)=1-4 \lambda^{-1} \log \lambda$. The right-hand side of (5.15) converges to $\frac{1}{2}$ as $\lambda \rightarrow \infty$, by virtue of Theorem 2.2, which is derived from Proposition 2.1 as we remarked above. We hence obtain Theorem 1.3.

\section{Proof of Theorem 1.4}

We first present a lemma in preparation for the proof of Theorem 1.4.

Lemma 6.1. Let $r$ be a real-valued function of $\lambda>0$ such that $r(\lambda) \rightarrow 1($ as $\lambda \rightarrow \infty)$. Then, for almost all $w \in \mathbb{W}$ (with respect to $\mathrm{P}$ ) and any $\varepsilon>0$,

$$
\lim _{\lambda \rightarrow \infty} \mathrm{P}_{\lambda w}^{0}\left\{\mathrm{e}^{\lambda(H-\varepsilon)} \leq \max _{0 \leq s \leq \mathrm{e}^{\lambda(\lambda)}} X(s) \leq \mathrm{e}^{\lambda(H+\varepsilon)}\right\}=1 .
$$

Proof. We prove that, for almost all $w \in \mathbb{W}$,

$$
\lim _{\lambda \rightarrow \infty} \mathrm{P}_{\lambda w}^{0}\left\{\tau\left(\mathrm{e}^{\lambda(H-\varepsilon)}\right)<\mathrm{e}^{\lambda r(\lambda)}<\tau\left(\mathrm{e}^{\lambda(H+\varepsilon)}\right)\right\}=1,
$$

which clearly implies the lemma. Let $w \in \mathbb{W}$ and, for any $\varepsilon$ such that $0<\varepsilon<H(w)$, let

$$
M^{\prime}= \begin{cases}\sigma\left(\frac{1}{2}-\varepsilon / 2\right) & \text { if } w \in A, \\ \sup \left\{x<\sigma\left(-\frac{1}{2}\right): w(x)-\min _{x \leq y \leq \sigma(-1 / 2)} w(y)=1-\varepsilon / 2\right\} & \text { if } w \in B .\end{cases}
$$

Then we see that

$$
\begin{aligned}
\lim _{\lambda \rightarrow \infty} \mathrm{P}_{\lambda w}^{0}\left\{\tau\left(\mathrm{e}^{\lambda(H-\varepsilon)}\right)<\tau\left(M^{\prime}\right)\right\} & =\lim _{\lambda \rightarrow \infty} \frac{\int_{M^{\prime}}^{0} \mathrm{e}^{\lambda w(x)} \mathrm{d} x}{\int_{M^{\prime}}^{0} \mathrm{e}^{\lambda w(x)} \mathrm{d} x+\mathrm{e}^{\lambda(H-\varepsilon)}} \\
& =1,
\end{aligned}
$$

since

$$
\lim _{\lambda \rightarrow \infty} \lambda^{-1} \log \int_{M^{\prime}}^{0} \mathrm{e}^{\lambda w(x)} \mathrm{d} x=\max _{M^{\prime} \leq x \leq 0} w(x) \geq H-\frac{\varepsilon}{2}>H-\varepsilon .
$$


Moreover, by applying Lemma 4.1 with $a=M^{\prime}$, we have

$$
\lim _{\lambda \rightarrow \infty} \mathrm{P}_{\lambda w}^{0}\left\{\tau\left(M^{\prime}\right)<\mathrm{e}^{\lambda r(\lambda)}\right\}=1
$$

Combining (6.2) and (6.3) yields

$$
\lim _{\lambda \rightarrow \infty} \mathrm{P}_{\lambda w}^{0}\left\{\tau\left(\mathrm{e}^{\lambda(H-\varepsilon)}\right)<\mathrm{e}^{\lambda r(\lambda)}\right\}=1 .
$$

Next, for any $\varepsilon>0$ we let

$$
M^{\prime \prime}= \begin{cases}\sigma\left(\frac{1}{2}+\varepsilon / 2\right) & \text { if } w \in A, \\ \sup \left\{x<\sigma\left(-\frac{1}{2}\right): w(x)-\min _{x \leq y \leq \sigma(-1 / 2)} w(y)=1+\varepsilon / 2\right\} & \text { if } w \in B .\end{cases}
$$

Then we have

$$
\begin{aligned}
\lim _{\lambda \rightarrow \infty} \mathrm{P}_{\lambda w}^{0}\left\{\tau\left(\mathrm{e}^{\lambda(H+\varepsilon)}\right)>\tau\left(M^{\prime \prime}\right)\right\} & =\lim _{\lambda \rightarrow \infty} \frac{\mathrm{e}^{\lambda(H+\varepsilon)}}{\int_{M^{\prime \prime}}^{0} \mathrm{e}^{\lambda w(x)} \mathrm{d} x+\mathrm{e}^{\lambda(H+\varepsilon)}} \\
& =1,
\end{aligned}
$$

since

$$
\lim _{\lambda \rightarrow \infty} \lambda^{-1} \log \int_{M^{\prime \prime}}^{0} \mathrm{e}^{\lambda w(x)} \mathrm{d} x=\max _{M^{\prime \prime} \leq x \leq 0} w(x) \leq H+\frac{\varepsilon}{2}<H+\varepsilon .
$$

Moreover, an application of Lemma 4.1 with $a=M^{\prime \prime}$ yields

$$
\lim _{\lambda \rightarrow \infty} \mathrm{P}_{\lambda w}^{0}\left\{\tau\left(M^{\prime \prime}\right)>\mathrm{e}^{\lambda r(\lambda)}\right\}=1
$$

By (6.5) and (6.6), we obtain

$$
\lim _{\lambda \rightarrow \infty} \mathrm{P}_{\lambda w}^{0}\left\{\mathrm{e}^{\lambda r(\lambda)}<\tau\left(\mathrm{e}^{\lambda(H+\varepsilon)}\right)\right\}=1
$$

which, combined with (6.4), proves (6.1). The proof of Lemma 6.1 is thus complete.

Proof of Theorem 1.4. By Lemma 2.1 and (1.2), we have

$$
\begin{aligned}
\int_{\mathbb{W}} \mathrm{P}(\mathrm{d} w) \mathrm{P}_{w}^{0}\left\{\left|\frac{\log \max _{0 \leq s \leq \mathrm{e}^{\lambda}} X(s)}{\lambda}-H\left(w_{\lambda}\right)\right|>\varepsilon\right\} \\
=\int_{\mathbb{W}} \mathrm{P}(\mathrm{d} w) \mathrm{P}_{\lambda w}^{0}\left\{\mid \frac{\left.2 \log \lambda+\log \max _{0 \leq s \leq \mathrm{e}^{\lambda r(\lambda)} X(s)}-H(w) \mid>\varepsilon\right\},}{\lambda}\right.
\end{aligned}
$$

where $r(\lambda)=1-4 \lambda^{-1} \log \lambda$. The right-hand side of (6.7) converges to 0 as $\lambda \rightarrow \infty$, by Lemma 6.1. We hence obtain Theorem 1.4.

\section{Acknowledgement}

The authors express their gratitude to Professor Hiroshi Tanaka for valuable suggestions and constant encouragement. 


\section{References}

[1] Brox, T. (1986). A one-dimensional diffusion process in a Wiener medium. Ann. Prob. 14, 1206-1218.

[2] Hu, Y. AND SHI, Z. (1998). The local time of simple random walk in random environment. J. Theoret. Prob. 11, 765-793.

[3] Itô, K. And McKean, H. P., JR. (1965). Diffusion Processes and their Sample Paths. Springer, Berlin.

[4] Kawazu, K., Suzuki, Y. and TanaKa, H. (2001). A diffusion process with a one-sided Brownian potential. Tokyo J. Math. 24, 211-229.

[5] Schumacher, S. (1985). Diffusions with random coefficients. In Particle Systems, Random Media and Large Deviations, ed. R. Durrett, American Mathematics Society, Providence, RI, pp. 351-356.

[6] SinaI, Ya. G. (1982). The limiting behavior of a one-dimensional random walk in a random medium. Theory Prob. Appl. 27, 256-268. 